vitro, Bergenin significantly increased the expression of ABCG2 as well as activated SIRT1, which was reversed by addition of PPARg antagonist GW9662 and siPPARg.

Conclusion: These findings suggest bergenin increases uric acid excretion both in the kidney and intestines, which may be related to the upregulation of ABCG2 via SIRT1- PPARg pathway.

References:

[1] Zhu, Y., Pandya, B. J. \& Choi, H. K. Prevalence of gout and hyperuricemia in the US general population: the National Health and Nutrition Examination Survey 2007-2008. Arthritis Rheum. 63, 3136-3141 (2011).

[2] Joosten LAB, Crisan TO, Bjornstad P, Johnson RJ: Asymptomatic hyperuricaemia: a silent activator of the innate immune system. Nature reviews Rheumatology 2020, 16(2):75-86.

Disclosure of Interests: None declared

DOI: 10.1136/annrheumdis-2020-eular.4163

\section{AB0054 \\ SYNOVIAL CD163+ MACROPHAGES ARE ASSOCIATED WITH RADIOGRAPHIC JOINT DESTRUCTION IN RHEUMATOID ARTHRITIS}

X. Zhang ${ }^{1}$, J. D. Ma ${ }^{1}$, J. Jing ${ }^{1}$, J. Wang ${ }^{1}$, T. Wu ${ }^{1}$, D. Zheng ${ }^{1}$, L. Dai ${ }^{2} .{ }^{1}$ Sun Yat-Sen Memorial Hospital, Sun Yat-Sen University, Guangzhou, China; ${ }^{2}$ Sun Yat-Sen Memorial Hospital, Sun Yat-Sen University, Guangzhou, China

Background: CD163, a hemoglobin scavenger receptor, has been identified as a marker of M2 macrophages, it can promote the release of IL-10 and carbon oxide. Researches on inflammatory diseases and tumors have suggested that CD163 plays anti-inflammatory effect and promotes tumor growth and metastasis. Rheumatoid arthritis (RA) is a chronic inflammatory disease characterized by chronic synovitis with inflammatory cells infiltration including considerable macrophages. However, little is known about the role of CD163+ macrophages in RA synovium.

Objectives: To investigate the expression and clinical significance of synovial CD163+ macrophages in RA.

Methods: Seventy-five RA patients were recruited and clinical data including disease activity, HAQ and Sharp/van der Heijde-modified Sharp score of bilateral hands and wrists were collected. Synovial tissues were obtained by needle biopsies or arthroscopy of knee joints. Eighteen osteoarthritis (OA) and seventeen orthopedic arthropathies (orth.A) patients were included as controls. All synovium were stained with $\mathrm{H} \& \mathrm{E}$ and immunohistochemically for CD163, CD3, CD20, CD38, CD68, and CD15. Histologic changes of synovitis in H\&E stained sections were graded with Krenn's synovitis score.

Results:

1. Positive CD163 expression were found in both lining synoviocytes and sublining inflammatory cells. Both densities of lining and sublining CD163+ macrophages in RA synovium were significantly higher than that in $\mathrm{OA}$ or Orth.A synovium $(140.47 \pm 66.93$ vs. $17.85 \pm 7.70$ vs. $19.76 \pm 5.26$ and $417.92 \pm 249.62$ vs. $27.58 \pm 14.19$ vs. $29.87 \pm 9.33$, all $P<0.001$, Figure 1)

2. According to Krenn's synovitis score, there were $68 \%$ RA patients showing high synovitis (score $>4)$. Both lining and sublining synovial CD163+ macrophages were significantly higher than those showing low synovitis (lining: $158.40 \pm 62.91$ vs. $122.06 \pm 66.74$, sublining: $462.96 \pm 62.91$ vs. $371.65 \pm 271.54$, both $P<0.05)$. Meanwhile, the densities of lining and sublining CD163+ macrophages were both positively correlated with Krenn's synovitis score $(r=0.238$ and 0.343 , both $P<0.05)$.

3. For clinical relationship in RA, the density of sublining CD163+ macrophages was positively correlated with total Sharp score (mTSS) $(r=0.399, P<0.001)$, joint space narrowing subscore $(r=0.248, P=0.032)$ and joint erosion subscore $(r=0.457, P<0.001)$. While the density of lining CD163+ macrophages was positively correlated with mTSS $(r=0.319, P=0.005)$ and joint erosion subscore $(r=0.358, P=0.002)$. Meanwhile, the densities of sublining and lining CD68+ macrophages were also positively correlated with mTSS $(r=0.253$ and 0.242 , both $P<0.05$ ), of which the correlation was weaker than that of CD163+ macrophages (Figure 2). There were no significant correlation between the density of CD163+ macrophages and disease activity or HAQ (all $P>0.05$ ).

Conclusion: Synovial CD163+ macrophages are associated with radiographic joint destruction, which imply that CD163+ macrophages may play role in the pathogenisis of joint destruction in RA.

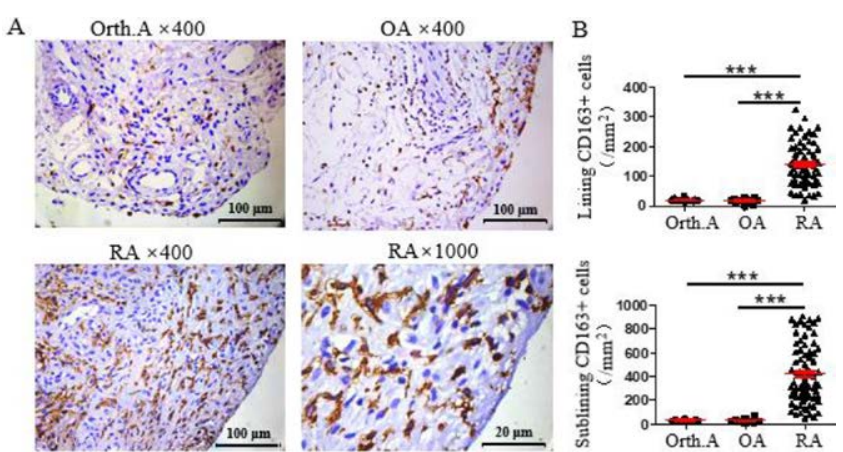

Figure 1. Representative immunohistochemical findings of synovial CD163 expression. (A) Synovial CD163 expression in an Orth.A patient, an OA patient and a RA patient. (B) Densities of lining and sublining CD163+ macrophages in Orth.A, OA and RA patients.
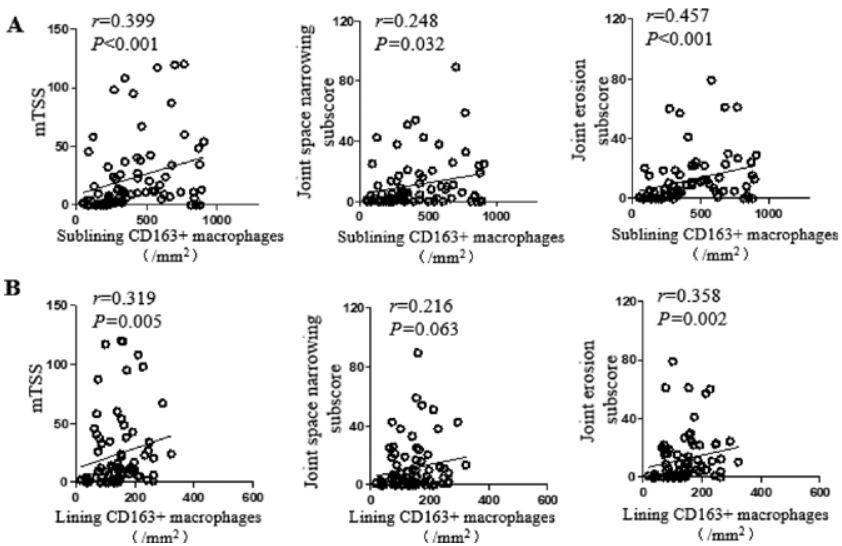

Figure 2. Spearman's rank correlation analysis for synovial macrophages and mTSS in RA (A) Correlation between sublining CD163+ macrophages and mTSS, joint space narrowing subscore, joint erosion subscore. (B) Correlation between lining CD163+ macrophages and mTSS, joint space narrowing subscore, joint erosion subscore.

Funding: : This work was supported by National Natural Science Foundation of China (no. 81801606 and 81971527), Guangdong Natural Science Foundation (no. 2017A030313576, 2018A030313541 and 2019A1515011928).

Figures:

Disclosure of Interests: None declared

DOI: 10.1136/annrheumdis-2020-eular.1141

\begin{tabular}{|l|l}
\hline AB0055 & SOLUBLE TREM-1 LEVELS IN FAMILIAL \\
MEDITERRANEAN FEVER RELATED \\
AA-AMYLOIDOSIS
\end{tabular}

S. Ugurlu', B. H. Egeli', I. M. Bolayirli', H. Ozdogan ${ }^{1} .{ }^{1}$ Istanbul University Cerrahpasa, Istanbul, Turkey

Background: Triggering Receptor Expressed on Myeloid cells-1 (TREM-1) is a monocyte and neutrophil receptor functioning in innate immunity. TREM-1 produces proinflammatory cytokines and serves for neutrophil degranulation. TREM-1 activity is well known in the pathogenesis of sepsis; hence it can be also present in autoinflammatory diseases such as the most common monogenic one, Familial Mediterranean Fever (FMF).

Objectives: The objective of this study is to measure soluble TREM-1 (sTREM1) activity in severe FMF cases complicated with systemic AA-Amyloidosis. Methods: The cohort of the study includes regularly followed FMF related AA-Amyloidosis patients in a tertiary center outpatient rheumatology clinic. Soluble TREM-1 levels were measured using enzyme-linked immunosorbent assay (ELISA). In addition, demographic data, renal function tests, acute phase reactants, and medical prescription history was also noted and analyzed. None of the FMF diagnosed patients had an attack during the collection of the blood samples. Results: The patients were categorized into 4 groups: FMF related AA-Amyloidosis patients $(A(+) F M F(+))$, FMF unrelated AA-Amyloidosis (FMF(-) $A(+))$, 